\title{
Stroke as a Presenting Feature of COVID-19
}

\author{
Asnin Shah, Niaz Ahmed Shaikh, Khalid Alattar, Liza Mariam Thomas, Uzma Sabahat \\ Dubai Health Authority, Internal Medicine, Rashid Hospital, Dubai, UAE \\ Email: Khalid_alattar@hotmail.com
}

How to cite this paper: Shah, A., Shaikh, N.A., Alattar, K., Thomas, L.M. and Sabahat, U. (2020) Stroke as a Presenting Feature of COVID-19. International Journal of Clinical Medicine, 11, 743-749. https://doi.org/10.4236/ijcm.2020.1112055

Received: November 10, 2020

Accepted: November 30, 2020

Published: December 3, 2020

Copyright $\odot 2020$ by author(s) and Scientific Research Publishing Inc. This work is licensed under the Creative Commons Attribution International License (CC BY 4.0).

http://creativecommons.org/licenses/by/4.0/

\section{(c) (i) Open Access}

\begin{abstract}
Objective: To analyze the coronavirus disease-2019 (COVID-19) patients presenting with acute stroke by determining their clinical characteristics, hospitalization course and prognosis. The common and conventional stroke risk factors in these patients were assessed with the aim of determining the role and contribution of the COVID-19 infection to stroke pathogenesis. Methods: Retrospective observational study involving 24 patients from a single tertiary care center over a time period of three months. Risk factors such as Age, Hypertension, Diabetes Mellitus, smoking status and underlying cardiac history were analyzed. COVID-19 relevant laboratory and radiological data were documented. Results: $87.5 \%$ of patients had ischemic stroke, with $58.3 \%$ of total patients being younger than 55 years. An equal incidence of both Diabetes Mellitus and Hypertension (37.5\%) was identified. $29.2 \%$ were completely asymptomatic for COVID-19, of which $85.7 \%$ had no chest X-ray changes on admission. Eight patients (61.5\%) developed pneumonia during admission despite an initially normal chest X-ray. Conclusion: Patients without COVID-19 symptoms and with normal chest radiography presenting with stroke does not rule out a possible underlying COVID-19 infection. Such patients may be positive for the virus and may go on to develop pneumonia shortly after suffering from strokes. This could suggest that stroke in COVID-19 patients is a possible initial presenting feature and consequence of the inflammatory state triggered by the Severe Acute Respiratory Syndrome Coronavirus-2 (SARS-CoV-2) infection. It is imperative to analyze the association of COVID-19 and stroke, and to maintain a high index of suspicion of COVID-19 infection in stroke patients, to enhance early detection and reduce transmission.
\end{abstract}

\section{Keywords}

COVID-19, Coronavirus, Stroke, Hypercoagulable, UAE

\section{Introduction}

Stroke is a well-studied clinical entity and a significant cause of mortality and 
morbidity, with well-recognized risk factors contributing to its pathogenesis. The Coronavirus disease of 2019 (COVID-19) caused by Severe Acute Respiratory Syndrome-Coronavirus-2 (SARS-CoV-2) is primarily a respiratory illness but multisystem involvement is being increasingly reported, including neurological system. A spectrum of neurological symptoms in COVID-19 patients including dizziness, headache and impairment of taste and smell has been demonstrated, with $5.7 \%$ of patients with severe infection and $0.8 \%$ with mild disease reporting acute cerebrovascular accidents [1]. Case series from New York [2] and Dubai, United Arab Emirates [3] have also reported stroke as a presenting feature of COVID-19. We report another series from the same center in Dubai with a different perspective to carry forward our interest in COVID-19 patients presenting with acute stroke.

Through this study, we aim to analyze COVID-19 patients presenting with acute stroke by determining their clinical characteristics, hospitalization course and outcome, and to evaluate the contribution of the conventional risk factors in causing stroke in these patients.

\section{Methods}

\subsection{Study Design}

This was a retrospective observational study from a single tertiary center caring for COVID-19 patients. Data was collected from the electronic medical records system during July-September 2020. The study was comprised of 24 patients admitted with a diagnosis of acute stroke and found to be COVID-19 positive within 24-48 hours of admission; over a time period of three months (July-September, 2020). Patients were included on the basis of World Health Organization (WHO) case definition of COVID-19 [4] with laboratory confirmation of SARS-CoV-2 by real-time polymerase chain reaction (RT-PCR) of nasopharyngeal swabs or endotracheal aspirates. Excluded participants were COVID-19 positive patients who developed a stroke 48 hours or more after admission and stroke patients who underwent thrombolysis or thrombectomy shortly after presenting. Stroke was defined on the basis of clinical presentation and imaging studies such as computed tomography of the brain.

\subsection{Study Variables}

Risk factors such as Age, Hypertension, Diabetes Mellitus, smoking status and underlying cardiac history were analyzed to determine their contribution to stroke pathology. These risk factors in particular were selected as they are known to influence the occurrence of strokes in individuals. In addition to classic stroke symptoms, presence of any pre-existing COVID-19 features were also recorded. Laboratory data including markers of inflammation and pro-thrombotic state such as C-reactive protein (CRP), D-Dimer, Ferritin, Lactate Dehydrogenase $(\mathrm{LDH})$ and Liver enzymes, and radiological data were documented. COVID-19 related and stroke-related complications during hospital stay were analyzed to provide a better reflection of patient outcome. 


\section{Standard Protocol Approvals, Registrations and Patient Consents}

This study was carried out in Rashid Hospital, Dubai, UAE and approved by the Dubai Ethical and Research Committee.

\section{Data Access Statement}

The data underlying this research paper was obtained with the permission of the ethical committee of Dubai Health Authority and cannot be made openly available due to confidentiality reasons. All relevant data supporting the study can be found in this paper.

\section{Results}

Out of the 24 patients who presented with stroke, $87.5 \%$ were ischemic $(70.8 \%$ thrombotic and $16.6 \%$ embolic), while $12.5 \%$ were hemorrhagic strokes. The mean age was 53 years with a range of $30-77$ years. $58.3 \%$ of patients were younger than 55 years, but had at least one other known contributory factor for stroke. $66.6 \%$ had dyslipidemia, 37.5\% had hypertension and/or Diabetes Mellitus. Only one patient had no known stroke risk factors.

$58.3 \%$ of the patients had elevated Procalcitonin (PCT) and D-dimer, while $45.8 \%$ had a raised CRP and $25 \%$ had ferritin levels above $300 \mathrm{ng} / \mathrm{mL} .29 .2 \%$ of the patients presented solely with stroke features and reported no prior COVID-19 symptoms. $45.8 \%$ of the patients had abnormal chest $\mathrm{x}$-rays on admission, showing either consolidations or infiltrates. Eight patients, with normal chest $\mathrm{X}$-ray on admission developed pneumonia during admission. Average duration of hospital stay was 15 days with $75 \%$ of patients demonstrating improvement in clinical status and 4 patients died due to COVID-19 related complications.

Table 1 compares our study population in terms of socio-demographics and stroke risk factors. Amongst both sets of patients in the table, most patients were males from the Indian subcontinent and overweight. The main differences between the two groups of patients shown in the table was that patients who presented without initial pneumonia had much less past history of smoking and less

Table 1. Socio-demographics and risk factors.

\begin{tabular}{ccc}
\hline & $\begin{array}{c}\text { Pneumonia on initial CXR } \\
11 \text { total patients } \\
\text { (as a \% of pneumonia } \\
\text { patients) }\end{array}$ & $\begin{array}{c}\text { No Pnonia on initial CXR } \\
\text { (as a \% of patients } \\
\text { without pneumonia) }\end{array}$ \\
\hline $\begin{array}{c}\text { Mean Age } \\
\text { Sex }\end{array}$ & 56 years & 50 years \\
Male & $9(81.8 \%)$ & $12(92.3 \%)$ \\
Female & $2(18.2 \%)$ & $1(7.7 \%)$ \\
Nationality & & $10(76.9 \%)$ \\
From the Indian Subcontinent \\
(India/Pakistan/Bangladesh)
\end{tabular}




\section{Continued}

\begin{tabular}{ccc}
\hline Philippines & $2(18.2 \%)$ & $1(7.7 \%)$ \\
Other nationalities & $1(9.1 \%)$ & $2(15.4 \%)$ \\
Body mass index (BMI) less than 25 & $\mathbf{2 ( 1 8 . 2 \% )}$ & $\mathbf{6 ( 4 6 . 2 \% )}$ \\
BMI more than/equal to 25 & $\mathbf{9 ( 8 1 . 8 \% )}$ & $\mathbf{7 ( 5 3 . 8 \% )}$ \\
Diabetic & $6(54.5 \%)$ & $2(15.4 \%)$ \\
Hypertensive & $\mathbf{5 ( 4 5 . 5 \% )}$ & $\mathbf{6}(\mathbf{4 6 . 2 \% )})$ \\
Dyslipidemic & $8(72.7 \%)$ & $8(61.5 \%)$ \\
Ischemic heart disease patient & $2(18.2 \%)$ & $2(15.4 \%)$ \\
Current/Previous smoker & $1(9.1 \%)$ & $3(23.1 \%)$ \\
Never smoker & $4(36.4 \%)$ & $8(61.5 \%)$ \\
Previous stroke patient based on CT & $2(18.2 \%)$ & $5(38.5 \%)$ \\
No risk factors for stroke & 0 & $1(7.7 \%)$ \\
\hline
\end{tabular}

Table 2. The clinical characteristics, laboratory and radiological parameters of patients with and without pneumonia on admission.

\begin{tabular}{|c|c|c|}
\hline & $\begin{array}{c}\text { Pneumonia on initial CXR } \\
11 \text { total patients } \\
\text { (as a \% of pneumonia patients) }\end{array}$ & $\begin{array}{c}\text { No Pneumonia on initial CXR } \\
13 \text { total patients } \\
\text { (as a \% of patients without pneumonia) }\end{array}$ \\
\hline \multicolumn{3}{|l|}{ Most common symptoms prior to stroke } \\
\hline Fever & $4(36.4 \%)$ & $1(7.7 \%)$ \\
\hline Cough & $4(36.4 \%)$ & $1(7.7 \%)$ \\
\hline Dyspnea & $2(18.2 \%)$ & $1(7.7 \%)$ \\
\hline $\begin{array}{l}\text { No prior symptoms suggesting infection } \\
\text { (asymptomatic COVID-19) }\end{array}$ & $1(9.1 \%)$ & $6(46.2 \%)$ \\
\hline Treated with COVID-19 targeted therapy & $10(90.9 \%)$ & $7(53.8 \%)$ \\
\hline \multicolumn{3}{|c|}{ Chest X-Ray } \\
\hline $\begin{array}{l}\text { Abnormal CXR on admission } \\
\text { - had either infiltrations or consolidations }\end{array}$ & $11(100 \%)$ & 0 \\
\hline $\begin{array}{l}\text { New pneumonia developed during admission } \\
\text { - admitted with normal CXR }\end{array}$ & 0 & $8(61.5 \%)$ \\
\hline \multicolumn{3}{|c|}{ Laboratory Findings } \\
\hline Elevated white blood cells (WBCs) & $2(18.2 \%)$ & $2(15.4 \%)$ \\
\hline Elevated PCT & $7(63.6 \%)$ & $7(53.8 \%)$ \\
\hline Elevated CRP & $7(63.6 \%)$ & $4(30.8 \%)$ \\
\hline High platelet count & $1(9.1 \%)$ & 0 \\
\hline Low platelet count & 0 & $2(15.4 \%)$ \\
\hline Elevated ferritin & $3(27.3 \%)$ & $3(23.1 \%)$ \\
\hline Elevated D-dimer & $6(54.5 \%)$ & $8(61.5 \%)$ \\
\hline Elevated prothrombin time (PT) & $11(100 \%)$ & $12(92.3 \%)$ \\
\hline Elevated activated partial thromboplastin time (APTT) & $1(9.1 \%)$ & $1(7.7 \%)$ \\
\hline International normalized ratio (INR) & $3(27.3 \%)$ & $4(30.8 \%)$ \\
\hline
\end{tabular}


diabetes. Table 2 compares the clinical characteristics, laboratory and radiological parameters of our study population. Many of the participants between both groups had elevated inflammatory markers and almost every single patient had an elevated prothrombin time. Additionally, the majority of patients who had pneumonia on presentation gave prior history of symptoms suggesting possible infection. Furthermore, even though most of our patients did not present with pneumonia on initial chest X-ray, more than half of these patients went on to develop pneumonia during hospital admission.

\section{Discussion}

Inflammatory conditions including acute respiratory infections are well-recognized triggers of cardiovascular events and stroke, and SARS-CoV-2 may act as a precipitating factor producing a pro-coagulant state either by inflammation, inducing a state of dehydration, development of antiphospholipid antibodies or due to large vessel vasculitis [5] [6].

Cardiovascular and cerebrovascular diseases share the same set of risk factors, and are important prognostic factors for poor outcome in COVID-19 infections [7]. South Asians have a higher risk of stroke compared to Whites with a younger age of onset and higher incidence of atherosclerotic risk factors such as $\mathrm{Di}$ abetes Mellitus and Hypertension [8]. Hypertension is also one of the five risk factors accounting for more than $80 \%$ of the global risk of all strokes [9]. Only $38 \%$ of our patients were known to be hypertensive, with all except one taking anti-hypertensives. The absence of lacunar infarcts (87\%), small vessel changes (71\%) or features of left ventricular hypertrophy on electrocardiogram (EKG) (87.5\%) in the rest of the patients suggest the absence of chronic undiagnosed hypertension in these patients, that otherwise could have been considered as the underlying risk factor. Although age is one of the well-recognized conventional risk factors for stroke, more than half of the patients in our study were below the age of 55. Other relevant stroke risk factors such as Diabetes Mellitus and Dyslipidemia were noted in $37.5 \%$ and $66.6 \%$ of patients respectively.

Stroke-related symptoms such as motor weakness (83.3\%) and speech impairment $(70.8 \%)$ were the primary reason that led to our patients seeking medical attention, and SARS-CoV-2 was then diagnosed, in-hospital by RT-PCR testing. $85.7 \%$ of patients who were asymptomatic for COVID-19 had no chest $\mathrm{X}$-ray changes on admission vs $14.3 \%$ who had pneumonia on admission despite being asymptomatic, which supports the idea that COVID-19 patients could present with stroke, much earlier than respiratory involvement can be objectively demonstrated. $61.5 \%$ of patients who presented with a normal chest X-ray went on to develop Pneumonia during hospital stay, which strongly suggests that stroke in COVID-19 patients could be the consequence and the first feature of an inflammatory state triggered by SARS-CoV-2.

SARS-CoV-2 has also been strongly associated with increased risk of venous and arterial thrombosis [10]. The COVID-19 associated coagulopathy was well 
reflected in our patients by raised D-Dimer levels in $58.3 \%$ and a modest increase in Prothrombin time in $\mathbf{9 5 . 8 \%}$. Raised inflammatory markers such as CRP, Procalcitonin and Ferritin, question the presence of inflammatory cytokines and their role in disrupting the blood brain barrier and resulting in neuro-inflammatory states that can precipitate cerebrovascular accidents, altered mental status, ataxia and seizures [11]. More than half of our patients presented with a raised procalcitonin on admission, while elevated levels of CRP and Ferritin was noted in $45.8 \%$ and $25 \%$ of patients respectively.

Our study was limited by the sample size of 24 patients, as it limited the diversity of clinical characteristics to be studied. In addition, due to logistical reasons including infection control policies and transfer of patients to other hospitals, complete stroke workup including transthoracic echo and carotid Doppler ultrasound could not be done in our facility; which could have added to the accuracy of number of thrombotic vs embolic sources of stroke.

\section{Conclusion}

Stroke is a well-studied clinical entity with various established risk factors and conventional clinical picture. However, during this pandemic increasing numbers of patients with COVID-19 infection are presenting with stroke and who either have no conventional risk factor or have apparently well controlled risk factors. It is imperative to consider the neurological impact of SARS-CoV-2 and its potential as a stroke risk factor, as stroke may be the presenting feature of COVID-19.

\section{Conflicts of Interest}

The authors declare no conflicts of interest regarding the publication of this paper.

\section{References}

[1] Mao, L., Jin, H., Wang, M., et al. (2020) Neurologic Manifestations of Hospitalized Patients with Coronavirus Disease 2019 in Wuhan, China. JAMA Neurology, 77, 683-690. https://doi.org/10.1001/jamaneurol.2020.1127

[2] Fara, M., Stein, L., Skliut, M., Morgello, S., Fifi, J. and Dhamoon, M. (2020) Macrothrombosis and Stroke in Patients with Mild COVID-19 Infection. Journal of Thrombosis and Haemostasis, 18, 2031-2033. https://doi.org/10.1111/jth.14938

[3] Khan, M., Ibrahim, R., Siddiqi, S., et al. (2020) COVID-19 and Acute Ischemic Stroke-A Case Series from Dubai, UAE. International Journal of Stroke, 15, 699-700. https://doi.org/10.1177/1747493020938285

[4] World Health Organization (2020) WHO COVID-19 Case Definition. World Health Organization.

https://www.who.int/publications/i/item/WHO-2019-nCoV-Surveillance Case Def inition-2020.1

[5] Smeeth, L., Thomas, S., Hall, A., Hubbard, R., Farrington, P. and Vallance, P. (2004) Risk of Myocardial Infarction and Stroke after Acute Infection or Vaccination. NeW England Journal of Medicine, 351, 2611-2618. 
https://doi.org/10.1056/NEJMoa041747

[6] Warren-Gash, C., Blackburn, R., Whitaker, H., McMenamin, J. and Hayward, A. (2018) Laboratory-Confirmed Respiratory Infections as Triggers for Acute Myocardial Infarction and Stroke: A Self-Controlled Case Series Analysis of National Linked Datasets from Scotland. European Respiratory Journal, 51, 1-17. https://doi.org/10.1183/13993003.01794-2017

[7] Pranata, R., Huang, I., Lim, M., Wahjoepramono, E. and July, J. (2020) Impact of Cerebrovascular and Cardiovascular Diseases on Mortality and Severity of COVID19-Systematic Review, Meta-Analysis, and Meta-Regression. Journal of Stroke and Cerebrovascular Diseases, 29, 1-9.

https://doi.org/10.1016/j.jstrokecerebrovasdis.2020.104949

[8] Gunarathne, A., Patel, J., Gammon, B., Gill, P., Hughes, E. and Lip, G. (2009) Ischemic Stroke in South Asians. Stroke, 40, 415-423.

https://doi.org/10.1161/STROKEAHA.108.535724

[9] O’Donnell, M., Xavier, D., Liu, L., et al. (2010) Risk Factors for Ischaemic and Intracerebral Haemorrhagic Stroke in 22 Countries (the INTERSTROKE Study): A Case-Control Study. The Lancet, 376, 112-123. https://doi.org/10.1016/S0140-6736(10)60834-3

[10] Al-Ani, F., Chehade, S. and Lazo-Langner, A. (2020) Thrombosis Risk Associated with COVID-19 Infection. A Scoping Review. Thrombosis Research, 192, 152-160. https://doi.org/10.1016/j.thromres.2020.05.039

[11] Sheraton, M., Deo, N., Kashyap, R. and Surani, S. (2020) A Review of Neurological Complications of COVID-19. Cureus, 12, 1-12. https://doi.org/10.7759/cureus.8192 